\title{
Effects of FESS and additional fluticasone propionate nasal drops on psychological well-being in nasal polyposis with asthma
}

\author{
STEVEN NORDIN ${ }^{1}$, PETTER OLSSON $^{2}$, EBBA HEDÉN BLOMQVIST ${ }^{2}$, \\ PÄR STJÄRNE ${ }^{2,3} \&$ ANDERS EHNHAGE ${ }^{2}$ \\ for the NAF2S2 Study Group ${ }^{\dagger}$ \\ ${ }^{\dagger}$ Nasal polyposis and Asthma: effects of FESS and FPND; the Stockholm Study \\ ${ }^{1}$ Department of Psychology, Umeå University, Umeå, Sweden \\ ${ }^{2}$ Department of Clinical Science, Intervention and Technology, Division of \\ Otorhinolaryngology, Karolinska Institutet, Stockholm, Sweden \\ ${ }^{3}$ Centre for Allergy Research, Karolinska Institutet, Stockholm, Sweden
}

Running Head: FESS, fluticasone and well-being in nasal polyposis

Corresponding author:

Steven Nordin, Ph.D.

Department of Psychology

Umeå University

SE-90187 Umeå, Sweden

E-mail: steven.nordin@psy.umu.se 


\section{Abstract}

Conclusion: A combined therapy of fluticasone propionate nasal drops (FPND) and functional endoscopic sinus surgery (FESS) can improve quality of life (QoL). When compared with prior data, the results imply that a generic measure of psychological aspects of QoL may be better than measures of respiratory symptoms and clinical parameters to capture a patient's perception of the disease and its treatment.

Objective: To better understand effects of FPND and FESS on generic QoL.

Methods: Sixty nasal polyposis patients with concomitant asthma completed participation in a randomized, double-blind, placebo-controlled, 14-week study in which they responded to the General Well-Being Schedule (GWBS).

Results: GWBS scores (i) increased significantly after administration of FPND, independent of FESS (from lower than normal to normal), (ii) increased after FESS independent of FPND (from lower than normal to normal), and (iii) increased additively after FPND and FESS.

Keywords: Functional endoscopic sinus surgery, General Well-Being Schedule, quality of life, randomized controlled trial 


\section{Introduction}

Management of nasal polyposis (NP) includes reducing or eliminating the polyps, opening the nasal airways, improving or restoring the sense of smell, preventing polyp recurrence, and improving the patient's quality of life (QoL) [1,2]. A first step of management is medical treatment with intranasal and, if necessary, additive treatment with oral corticosteroids [3]. It is documented that administration with fluticasone propionate nasal drops (FPND) can decrease polyp size and nasal congestion [4,5]. Although it has been suggested that the effects of nasal polypectomy and functional endoscopic sinus surgery (FESS) on lower airways in NP is not conclusive [3], we recently reported reduced upper as well as lower airway symptoms and improvement in clinical parameters (PNIF, olfactory thresholds, PEFR) after FESS. However, we found no significant improvement after 4 weeks pre-operative treatment with FPND vs placebo, and no additional effect of FPND to the FESS effects [6].

Assessment of patient-reported outcome is important in clinical settings, and in some cases symptomatic outcome should be the primary treatment outcome [7]. In this respect measurement of health-related QoL plays an important role. A large number of studies have demonstrated poor health-related QoL in NP as well as in asthma with respect to both generic and specific aspects of QoL [7]. NP and asthma symptomology is likely to contribute to poor psychological well-being, of which important aspects include anxiety, mild depression and low vitality, making these patients particularly prone to improvement in psychological aspects of QoL [8]. Support for this has recently been reported from patients with NP and asthma, by assessment with the Short Form 36 (SF-36) [9]. In that study domains of psychological nature were particularly improved after FESS and administration of FPND. However, with this exception, there are no previous studies that, with convincing evidence, have focused exclusively on the health profile of NP with concomitant asthma. Thus, for further 
understanding of effects of FESS and FPND on NP and asthma, the study of psychological aspects of QoL seems to be particularly relevant.

An objective of the present study was to investigate the impact of FESS and FPND on psychological well-being by means of the General Well-Being Schedule in patients with NP and concomitant asthma. The GWBS is a generic instrument for assessing psychological wellbeing and distress, with the underlying dimensions anxiety, depression, general health, positive well-being, self-control and vitality $[10,11]$. Considering the suggested improvement in nasal symptoms after FPND [5] and improvement in airway symptoms and clinical parameters after FESS [6], it was hypothesized (i) that administration with $400 \mu \mathrm{g}$ b.i.d. of FPND prior to FESS would have a positive impact on psychological well-being in NP with asthma, (ii) that FESS without FPND administration also would have such an impact, and (iii) that FPND would provide an additional positive impact above that of FESS.

\section{Material and methods}

\section{Study design, treatment and patients}

This was a prospective, 14-week, single-center study in which all patients underwent FESS, and the topical medical treatment included a randomized, prospective, double-blind placebocontrolled design on FPND vs placebo. Asthma treatment was not to be changed during the study, and oral corticosteroid treatment, but not aspirin sensitivity, was an exclusion criterion. Aspirin sensitivity was not further investigated by provocation test or specific history. The study design is illustrated in Figure 1. At Visit 1 the patients were examined by an ENTphysician and a pulmonologist, confirming the diagnosis of NP and asthma. At Visit 2, after 4 weeks of washout from nasal steroid treatment and a CT scan verifying the diagnosis of NP, the patients were randomized to either $400 \mu \mathrm{g}$ b.i.d. treatment of FPND or to placebo for 10 weeks. Visit 3 was made four weeks after Visit 2. About one week after that, all patients 
underwent FESS, and within one week post-surgery, at Visit 4, all patients had post-surgical follow-up (nasal debridement). Thereafter they had 4 additional weeks of treatment with FPND/placebo until Visit 5. Consequently, the patients responded to the GWBS questionnaire at Visits 2 (after wash out), 3 (after FPND/placebo treatment) and 5 (after FESS and FPND/placebo treatment). All patients received FPND treatment after Visit 5 (until their last visit, Visit 6).

[Figure 1 about here]

Sixty-eight patients were randomized to one of the two groups (1:1). However, eight of the patients were not included (due to meeting exclusion criteria, $n=6$, and refusing further participation, $\mathrm{n}=2$ ). Thus 60 patients completed the study. Of these, 26 took part in the FPND group $($ mean \pm SD age $=51.0 \pm 14.6$ years $)$ and 34 in the placebo group $(51.8 \pm 14.3$ years). Mean polyp score $(\max 3)$ at baseline was 2.3 in the FPND group and 2.2 in the placebo group. Detailed patient characteristics are presented in Table I. For further description of study design, treatment and patients, see [6].

[Table I about here]

\section{General Well-Being Schedule}

In addition to questions from the Multi-Clinic Smell and Taste Questionnaire [12] pertaining to nasal and respiratory symptoms and prior nasal/sinus surgery (Table I), the patients responded to the GWBS $[10,11]$ under supervision at the ENT department. The GWBS consists of 18 items that indicate subjective feelings of psychological well-being and distress. The first 14 questions use a 6-point rating scale that represents either intensity or frequency, and the remaining four items use an 11-point rating scale that is anchored by adjectives. The score can vary from 0 to 110 (high score indicating positive well-being and low distress). The GWBS assesses the six latent dimensions anxiety, depression, general health, positive well- 
being, self-control and vitality. It has good internal consistency, test-retest reliability and validity [13], and has normative data for categories of well-being (positive well-being, moderate distress and severe distress) [14].

\section{Statistical methods}

Changes in GWBS score across visits were analyzed by applying two-way (visit $\times$ FPND group) mixed-model analyses of variance (2-tailed; Greenhouse-Geisser correction) between adjacent visits (Visit 2 vs Visit 3 and Visit 3 vs Visit 5). Further analyses of interactions were conducted with paired t-test (2-tailed), and an independent-sample t-test (2-tailed) was used to compare GWBS score between groups at Visit 2. For comparison with population-based normative data [14], the patients were categorized into the subgroups "positive well-being" (GWBS scores 73-110), "moderate distress" (scores 61-72) or "severe distress" (scores 0-60) for each visit. Comparisons of distribution across GWBS categories between the patient groups and normative data for each visit were conducted with chi-square analysis. The $\alpha$-level was set at 0.05, and SPSS 15.0 for Windows (SPSS Inc., Chicago, IL, USA) was used.

\section{Results}

Mean GWBS scores are presented in Figure 2. The two groups did not differ significantly in GWBS score at Visit $2(t=0.35, \mathrm{p}>0.72$; Figure 2). Regarding Visits 2 and 3, there was a main effect of visit $(F=11.05, \mathrm{p}<0.001)$ on GWBS score, and a visit $\times$ FPND group interaction $(F=4.13, p<0.05)$, but no main effect of FPND group $(F=1.35, p>0.25)$. Further analysis of the interaction showed a significant increase in GWBS score from Visit 2 to 3 in the FPND group $(t=4.07, p<0.001)$ but not in the placebo group $(t=0.90, p>0.37)$. Regarding Visits 3 and 5, there were main effects of both visit $(F=16.34, p<0.001)$ and FPND group $(\mathrm{F}=4.59, \mathrm{p}<0.05)$ on GWBS scores, but no visit $\times$ FPND group interaction $(\mathrm{F}$ 
$=0.08, \mathrm{p}>0.78)$. These results were verified by a significant increase in GWBS score from Visit 3 to 5 in both the FPND group $(\mathrm{t}=2.56, \mathrm{p}<0.05)$ and placebo group $(\mathrm{t}=3.22, \mathrm{p}<$ 0.01). Thus, (i) whereas the two groups were similar at baseline after wash-out (Visit 2), (ii) the FPND group, but not the placebo group, increased significantly in GWBS score 4 weeks post-FPND onset pre-surgery (Visit 3), and (iii) both groups increased in GWBS score to a similar extent post-surgery (Visit 5).

The distributions across the three categories of well-being and distress for the patients and for the normative data are presented in Figure 3. At Visit 2 both the FPND and placebo group differed significantly in distribution from that of normative data, with a larger proportion of the patients being categorized as distressed (Figure 3). At Visit 3 the placebo group remained significantly different from normative data, whereas the FPND group did no longer differ significantly. At Visit 5 none of the two groups differed significantly from normative data. Thus, (i) a larger proportion of the patients in both groups, compared to normality, showed distress at baseline after wash-out (Visit 2), (ii) the FPND group but not placebo group had shifted to normality 4 weeks post-FPND onset pre-surgery (Visit 3), and (iii) post-surgery (Visit 5) the placebo group had also shifted to normality, and the FPND group remained normal in well-being and distress.

[Figures 2 and 3 about here]

\section{Discussion}

The outcome of this randomized, prospective, double-blind, placebo-controlled study, one of few including a homogenous group of NP with concomitant asthma, supports the hypotheses (i) that 4 weeks of administration with $400 \mu \mathrm{g}$ b.i.d. of FPND has a positive impact on psychological well-being in patients with NP with asthma, (ii) that FESS without FPND administration also has such an impact, and (iii) that FPND provides an additional positive 
impact above that of FESS. The categorization of GWBS score and comparison with population-based normative data further suggest that psychological well-being in this patient population is poorer than in the general population, and, importantly, that their well-being reaches the level of the general population after only 4 weeks of FPND administration. The results further suggest that FESS stand alone results in a normal level of well-being. These positive effects of both nasal corticosteroid treatment and FESS are consistent with findings of improved QoL assessed with the SF-36 [8,9].

The improvement after FPND administration is in contrast to our earlier data with measurements of self-reported airway symptoms and clinical parameters [6]. It is quite possible that measures of generic QoL that are based on a sum score of a large number of items, such as the GWBS, are more sensitive than individual symptom ratings in capturing the patient's perception of the disease and its treatment. However, as for any generic measure of QoL, it cannot be taken for granted that the improvement in psychological well-being can fully be accounted for by the medical and surgical treatment under study.

In assuming that the present measures of QoL can be referred predominantly to the $\mathrm{NP} /$ asthma condition, it is difficult to more specifically determine to what extent poor QoL is caused by the different components of the condition: the nasal polyps, the asthma or an olfactory loss that is secondary to the polyps and asthma. QoL has been reported to be poorer in NP with asthma than in NP without asthma $[15,16]$. Thus, NP and asthma seem to have a cumulative negative effect on QoL [17]. However, QoL in patients with isolated asthma has been shown to be better than in isolated NP, suggesting that NP impairs QoL to a larger extent than asthma [15]. A majority of the current patients had a loss in odor sensitivity, and it is well documented that such a loss is associated with poor QoL in general [18], but also with respect to psychological well-being and specific aspects of QoL assessed as in the present study [19]. Taken together, NP may account for the poor QoL to a larger extent than both 
asthma and olfactory loss. Another limitation is that the randomized, double-blinded, controlled part of this study lasted for only 5 weeks after FESS, so no double-blinded and controlled data are available beyond this period. Thus, it is not possible to tell from the present data for how long the well-being in these patients remained at a normal level.

To conclude, the results from this randomized, prospective, double-blind, placebocontrolled study suggests that psychological well-being is poorer than normal in NP with asthma, but that FPND administration stand alone and FESS stand alone improve general well-being to a normal level. An implication of these results is that QoL may preferably be evaluated in NP patients with asthma.

\section{Ackowledgements}

Financial support for this study was provided by GlaxoSmithKline, the Swedish Association of Otorhinolaryngology, Head and Neck Surgery, the Acta Otolaryngologica Foundation, and the Swedish Asthma and Allergy Association. We wish to thank Vania Sandberg, Agneta Gülich, Ann-Sofie Lantz, Christa Elgerius, Marianne Eduards, Katarina Söderström, KarlGustav Kölbeck, Maria Skedinger, Barbro Dahlén, Gert Henriksson, Peter Graf and Karin Toll for their help with this study.

Declaration of Conflicting Interests: The authors declare that there is no conflict of interest. The authors alone are responsible for the content and the writing of this paper. After the end of this study Dr. Olsson was employed by MSD, Sweden, and is presently employed by Boehringer-Ingelheim, Sweden. 


\section{References}

[1] Mygind N. Advances in the medical treatment of nasal polyps. Allergy 1999; 54(Suppl 53):12-16.

[2] Tuncer U, Soylu L, Aydogan B, Karakus F, Akcali C. The effectiveness of steroid treatment in nasal polyposis. Auris Nasus Larynx 2003;30:263-268.

[3] Fokkens W, Lund V, Mullol, J. EP3OS 2007. European position paper on rhinosinusitis and nasal polyps 2007. A summary for otorhinolaryngologists. Rhinol 2007; 45:97-101.

[4] Holmström M. Clinical performance of fluticasone propionate nasal drops. Allergy 1999; 54(Suppl 53):21-25.

[5] Penttilä M, Poulsen P, Hollingworth K, Holmström M. Dose-related efficacy and tolerability of fluticasone propionate nasal drops 400 microg once daily and twice daily in the treatment of bilateral nasal polyposis: a placebo-controlled randomized study in adult patients. Clin Exp Allergy 2000; 30:94-102.

[6] Ehnhage A, Olsson P, Kölbeck KG, Skedinger M, Dahlén B, Ålenius M, Stjärne P. Functional endoscopic sinus surgery improved asthma symptoms as well as PEFR and olfaction in patients with nasal polyposis. Allergy 2009; 64:762-769.

[7] Alobid I, Bernal-Sprekelsen M, Mullol, J. Chronic rhinosinusitis and nasal polyps: the role of generic and specific questionnaires on assessing its impact on patient's quality of life. Allergy 2008; 63:1267-1279.

[8] Alobid I, Benítez P, Bernal-Sprekelsen M, Guilemany JM, Picado C, Mullol J. Nasal polyposis and its impact on quality of life: comparison between the effects of medical and surgical treatments. Allergy 2005; 60:452-458.

[9] Olsson P, Ehnhage A. Nordin S, Stjärne, P. Quality of life is improved by endoscopic sinus surgery and fluticasone nasal drops in nasal polyposis with asthma. Rhinology, in press. 
[10] Dupuy HJ. Self-representations of general psychological well-being of American adults. Paper presented at American Public Health Association Meeting, Los Angelses, October, 1978.

[11] McDowell I: Measuring health: a guide to rating scales and questionnaires. New York: Oxford University Press, 2006.

[12] Nordin S, Brämerson A, Murphy C, Bende M. A Scandinavian adaptation of the MultiClinic Smell and Taste Questionnaire: evaluation of questions about olfaction. Acta Otolaryngol 2003; 123:536-542.

[13] Fazio AF. A concurrent validational study of the NCHS General Well-Being Schedule. Vital Health Stat 2 1977; 73:1-53.

[14] Bowling A. Measuring health: a review of quality of life measurement scales. Philadelphia: Open University Press, 1997.

[15] Bousquet J, Bullinger M, Fayol C, Marquis P, Valentin B, Burtin B. Assessment of quality of life in patients with perennial allergic rhinitis with the French version of the SF-36 Health Status Questionnaire. J Allergy Clin Immunol 1994; 94:182-188.

[16] Bousquet J, Knani J, Dhivert H, Richard A, Chicoye A, Ware JE Jr, Michel FB. Quality of life in asthma. I. Internal consistency and validity of the SF-36 questionnaire. Am J Respir Crit Care Med 1994; 149:371-375.

[17] Radenne F, Lamblin C, Vandezande LM, Tillie-Leblond I, Darras J, Tonnel AB, Wallaert B. Quality of life in nasal polyposis. J Allergy Clin Immunol 1999; 104:79-84.

[18] Hummel T, Nordin S. Olfactory disorders and their consequences for quality of life: a review. Acta Otolaryngol 2005; 125:116-121.

[19] Hedén Blomqvist E, Brämerson A, Stjärne P, Nordin S. Consequences of olfactory loss and adopted coping startegies. Rhinol 2004; 42:189-194. 


\section{Figure legends}

Figure 1. Study design. Patients were evaluated at Visit 1 (v1) by an ENT physician and a pulmonologist, and were at Visit 2 (v2) randomized to either FPND treatment or placebo. Prior to surgery (FESS), patients were examined at Visit 3 (v3) with postsurgical follow-up (nasal debridement) at Visit 4 (v4). All patients received FPND treatment after Visit 5 (v5) until their last visit, Visit 6 (v6). Adopted from [6] with permission from Wiley.

Figure 2. Mean \pm SE score on the General Well-Being Schedule in the fluticasone propionate nasal drop (FPND) group $(\mathrm{n}=26)$ and placebo group $(\mathrm{n}=34)$ at Visits $2(\mathrm{v} 2), 3(\mathrm{v} 3)$ and 5 (v5).

Figure 3. Proportions of patients in the fluticasone propionate nasal drop (FPND) group (n= 26) and placebo group $(n=34)$ who are referred to different categories based on score on the General Well-Being Schedule at Visits 2 (v2), 3 (v3) and 5 (v5). Statistical significance levels are given for distribution comparisons with normative data [14] based on chi-square analyses. 
Figure 1

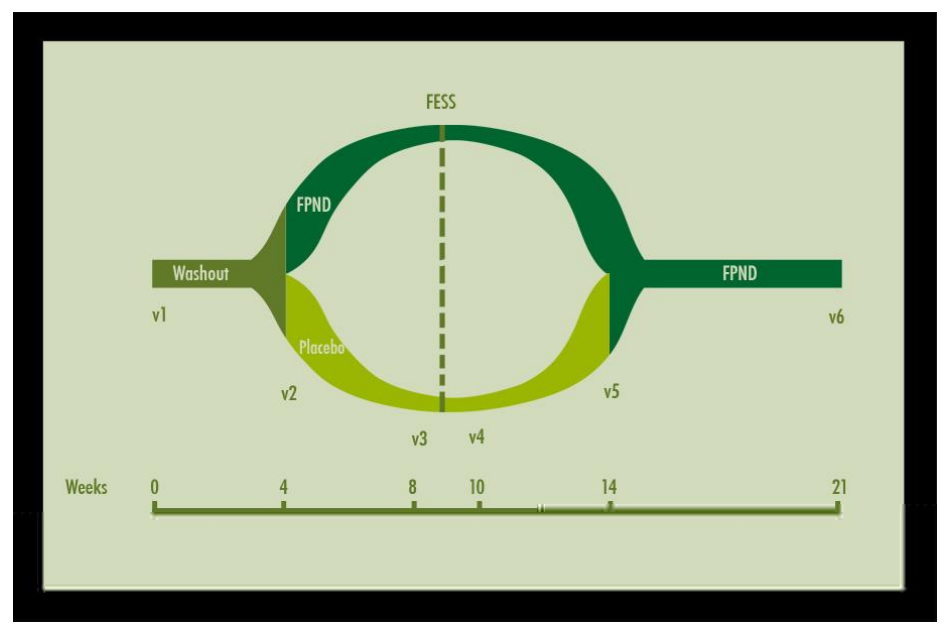


Figur 2

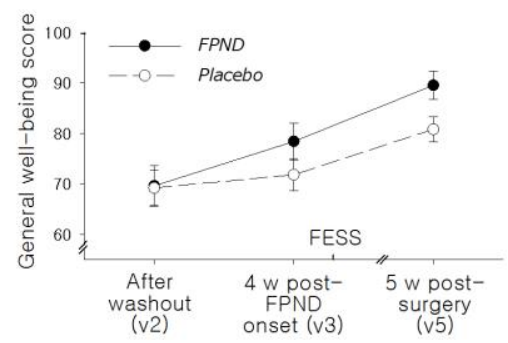


Figure 3

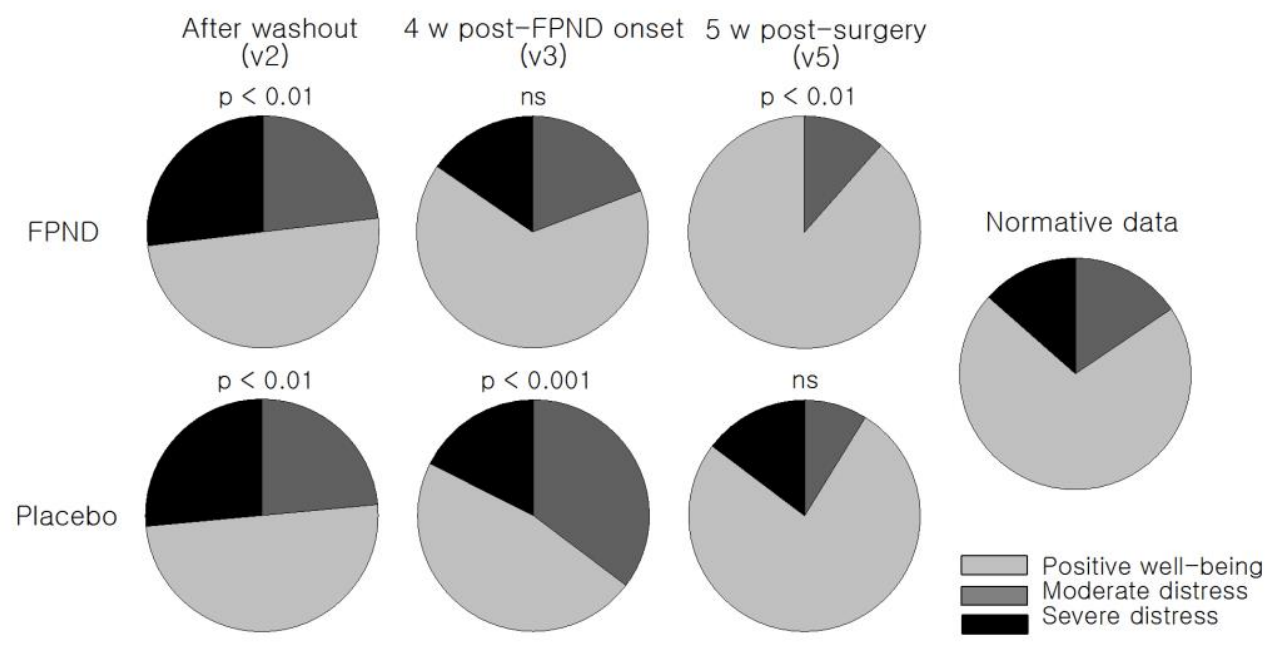

